\title{
Materials and techniques used for the "Vienna Moamin": multianalytical investigation of a book about hunting with falcons from the thirteenth century
}

\author{
Wilfried Vetter ${ }^{*}(\mathbb{D}$, Bernadette Frühmann, Federica Cappa and Manfred Schreiner
}

\begin{abstract}
A multianalytical approach was used to characterize the materials in the "Vienna Moamin", an outstanding richly illustrated manuscript from the late thirteenth century, which was made in Italy and is now kept in the Kunsthistorisches Museum Wien. The investigations were carried out with a non-invasive approach by using complementary techniques, such as X-ray fluorescence (XRF), reflection Fourier transform infrared spectroscopy (rFTIR), Raman spectroscopy, and fiber optic reflectance spectroscopy (FORS). In addition, XRF scans were performed in two areas which yielded chemical maps showing the elemental distribution. The results revealed that typical materials from the medieval times were applied for the manuscript. Calcium carbonate on the parchment surface indicated a dehairing process with lime and/or whitening with chalk. Two different iron gall inks were detected in the main text and marginal notes, and vermilion was used for rubrication. The color palette included azurite, a green colorant composed of orpiment and indigo, yellow ochre, brown iron oxide pigments, minium, vermilion, brazilwood lake, and carbon black. Moreover, mosaic gold was detected in gold-beige hues. Lead white was identified for white areas and fine decoration lines, as well as in mixture with blue and red pigments for light color shades. No reliable information could be obtained concerning the binding media. Two differing application techniques for gold leaf were detected, which correspond with stylistic differences: either on gypsum or chalk preparation layers. Furthermore, calcium soap contents in certain colors were determined only on one folio with unique characteristics. The XRF scans of two historiated initials revealed that similar materials were applied in both cases and provided further valuable information about the painting technique. The results obtained enabled to gain insights into Italian thirteenth century manuscript production techniques and to characterize the used materials. The investigations showed the importance of scanning XRF for the elucidation of painting techniques, but also the demand of scanning devices utilizing compound specific analytical techniques such as rFTIR.
\end{abstract}

Keywords: Illuminated manuscripts, Non-invasive, Material analyses, Painting technique, Scanning XRF, Reflection FTIR, Raman, FORS, Mosaic gold, Brazilwood lake

*Correspondence: w.vetter@akbild.ac.at

Institute of Science and Technology in Art, Academy of Fine Arts Vienna, Schillerplatz 3, 1010 Vienna, Austria

\section{Introduction}

In the year 1240 Emperor Frederick II of Hohenstaufen (1194-1250), a great enthusiast of falconry and author of an unrivalled treatise on the subject, "De arte venandi cum avibus", ordered the translation into Latin of an Arabic treatise based on texts of the eighth and ninth century. His court philosopher Theodore of Antioch
袋 Springer Open

c) The Author(s) 2021. This article is licensed under a Creative Commons Attribution 4.0 International License, which permits use, sharing, adaptation, distribution and reproduction in any medium or format, as long as you give appropriate credit to the original author(s) and the source, provide a link to the Creative Commons licence, and indicate if changes were made. The images or other third party material in this article are included in the article's Creative Commons licence, unless indicated otherwise in a credit line to the material. If material is not included in the article's Creative Commons licence and your intended use is not permitted by statutory regulation or exceeds the permitted use, you will need to obtain permission directly from the copyright holder. To view a copy of this licence, visit http://creativeco mmons.org/licenses/by/4.0/. The Creative Commons Public Domain Dedication waiver (http://creativecommons.org/publicdomain/ zero/1.0/) applies to the data made available in this article, unless otherwise stated in a credit line to the data. 
thus compiled the "Liber Moamin falconarii de scientia venandi per aves et quadrupedes" (The Book of Falconer Moamin on the Art of Hunting with Birds and Quadrupeds), which was personally revised by Frederick II during the siege of Faenza [1]. The Latin text became famous and was copied multiple times, and it was also translated into Neapolitan, Tuscan and Franco-Italian [2]. The "Vienna Moamin", a luxury volume for a high ranking person, is one of the 29 surviving Latin copies, and dates back to circa 1300. At the middle of the fifteenth century it was in Hungary, in the hands of Janos Rozgonyi, member of the royal court, before being acquired by a Viennese humanist and physician, Johannes Fuxmagen who wrote his name in the volume. At the beginning of the sixteenth century the manuscript was part of the collection of Emperor Maximilian I (1459-1519), a passionate hunter himself, and later on it passed to Archduke Ferdinand II. of Tyrol (1529-1595) [2]. It is nowadays kept in the Imperial Armoury (Hofjagd- und Rüstkammer), Kunsthistorisches Museum Wien (Inv. No. K4984). The manuscript was made in central or southern Italy by presently unknown artists and consists of 54 parchment folios with single column text and 101 historiated initials. Interestingly, instructions in Italian for the illuminator were written in the margins and have survived for the most part, giving an insight into the creation of the scenes [3]. As an example, folio 47 verso is presented in Fig. 1. The parchment and the text of the manuscript are relatively well preserved. The same accounts for the majority of the illuminations, although delamination of gold leaf and localized paint losses occurred frequently. Extensive media loss must be observed only on few folios, including folio $1 \mathrm{r}$ which was investigated in this study. It represents a document of high cultural and historical significance, which contains an in-depth treatment of the species of birds of prey used in falconry and the medical treatment of their illnesses, as well as those of hunting dogs. A real gold and a standard facsimile edition have been brought out recently [4]. Moreover, a commentary volume including codicological information is presently in press [5].

Progresses in the design of mobile analytical instruments, which enable to characterize materials in manuscripts non-invasively in libraries and museums, led to an increased collaboration between natural sciences and the humanities, particularly material sciences and philology. A common interest concerns the question if the results from material analyses can be used to attribute manuscripts to particular authors or workshops. This seems basically possible, as the materials in medieval times often contained impurities or characteristic trace elements. Moreover, the application techniques were not standardized and specific practices of certain workshops could provide useful information for an attribution.
However, it is not as simple in practice. An unambiguous attribution requires ideally complete data sets containing the chemical and historical information about local workshops/persons over long time periods, which may serve as references for the particular styles. For this reason, one aim of the Centre of Image and Material Analysis in Cultural Heritage in Vienna (CIMA) [6] is to build corresponding databases as complete as possible and to link it with other institutions in a further step.

To the best of our knowledge, only few chemical data has been published concerning the materials in late thirteenth century manuscripts from Italy [7]. Hence, the results reported in this study may add to the knowledge about medieval manuscript production and provide reference data for the attribution of other manuscripts. Some obstacles which may complicate an attribution, e.g. the contribution of multiple artists, have been mentioned by Trentelman and Turner [8] and will be addressed as well in the present study. Moreover, the strengths and weaknesses of the applied analytical techniques will be discussed. Particular attention will be given to XRF scanning, which has been increasingly used in combination with other techniques for the study of manuscripts in the last years [9-12].

\section{Experimental}

During two measurement campaigns the Vienna Moamin was investigated at the Academy of Fine Arts Vienna (2017) and the conservation workshop of the Collection of Arms and Armour, Kunsthistorisches Museum Wien (2018) by using mobile equipment for completely noninvasive analyses. The applied techniques were X-ray fluorescence (XRF), Raman spectroscopy, reflection Fourier transform infrared spectroscopy (rFTIR), and fiber optic reflectance spectroscopy (FORS).

During the analyses the manuscript was placed horizontally on a lifting platform, which was used for adjusting the measuring position and distance. A felt below the lifting platform facilitated its horizontal movement. The instruments were mounted either on a tripod for the XRF and Raman analyses, or a tetrapod in case of rFTIR and FORS as shown in Fig. 2. The tetrapod provides maximum stability, particularly if the rFTIR instrument weighing ca. $8 \mathrm{~kg}$ is mounted, and can be disassembled for easy transport.

Prior to the analyses, 21 folios were chosen after visual inspection of the manuscript with the intention to cover as many analytical questions regarding materials and techniques as possible. Due to the limited access to the manuscript, not all measurement points were analyzed by all methods available. The analyzed folios are listed in Table 1. In total 186 measurement points (mp) were analyzed. XRF was applied on 21 folios (171 


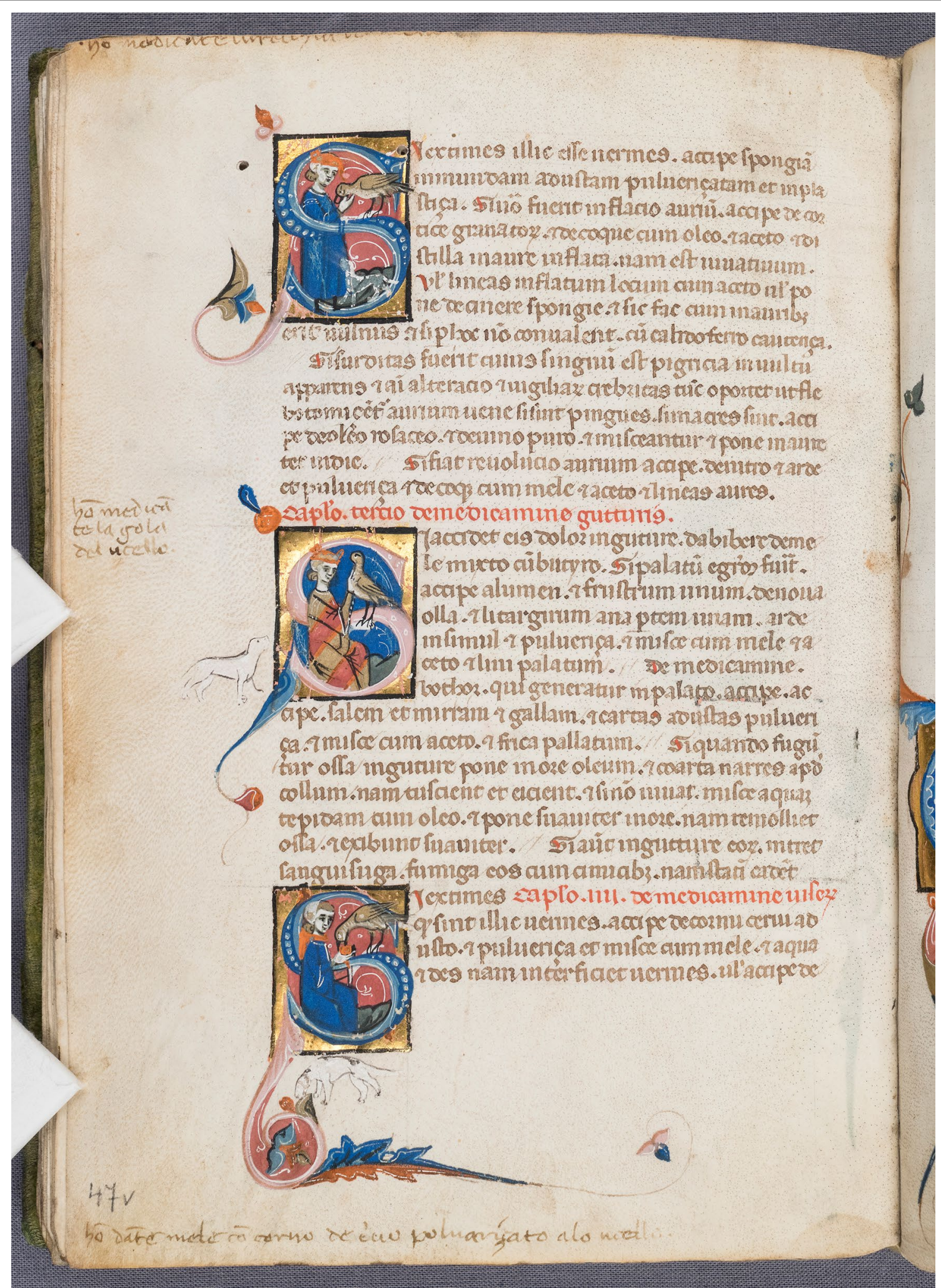

Fig. 1 Visible light image of folio 47v in the Vienna Moamin (thirteenth century) 


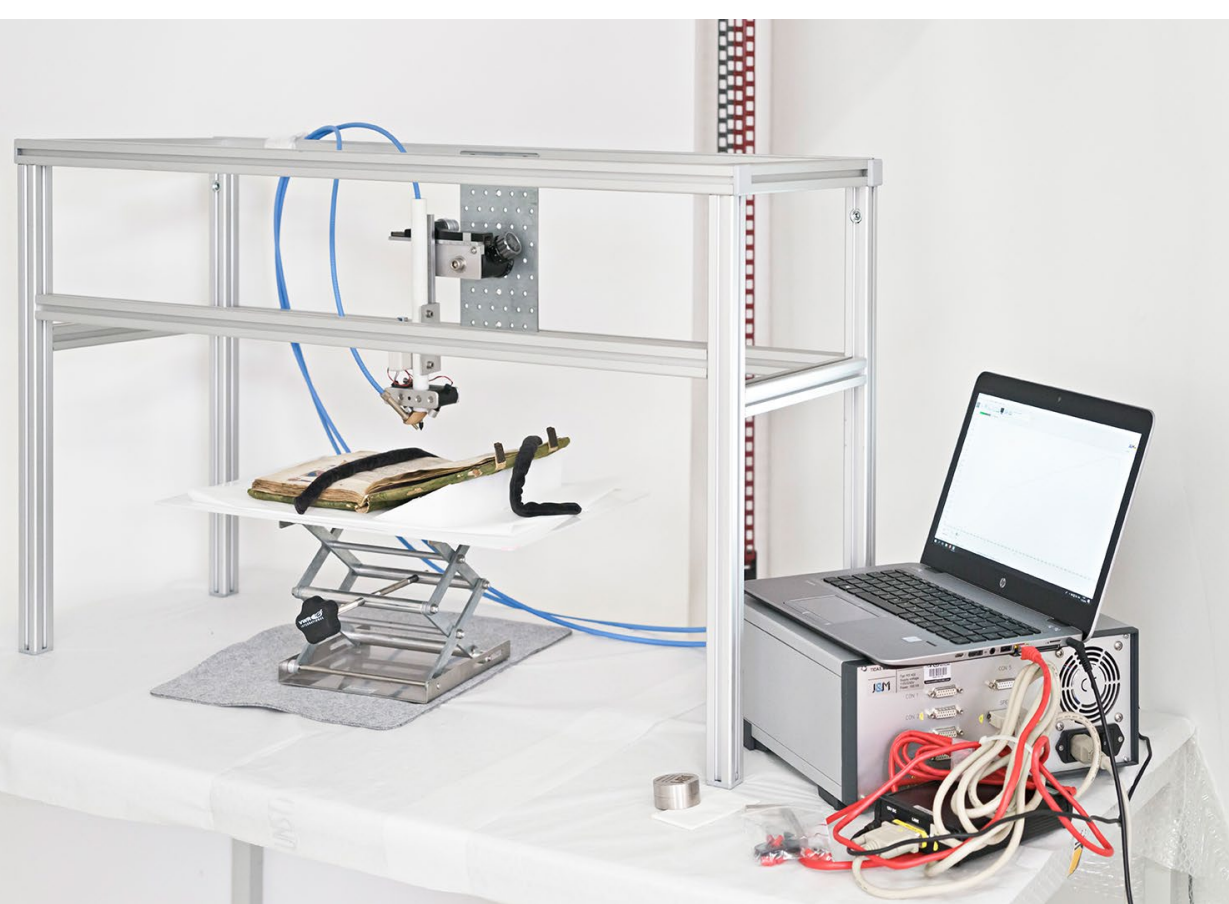

Fig. 2 FORS analyses of the Vienna Moamin. A lifting platform was used for the adjustment of the measuring position and the adequate distance. The fiber optic probe was mounted on a tetrapod, which also served to mount the rFTIR instrument

Table 1 List of folios analyzed in the Vienna Moamin

\begin{tabular}{|c|c|c|c|c|c|c|c|c|c|c|c|c|c|}
\hline \multirow[t]{2}{*}{ Technique } & \multicolumn{13}{|l|}{ Folio } \\
\hline & $1 \mathrm{r}$ & $1 \mathrm{v}$ & $3 r$ & $3 v$ & & $10 v$ & & $16 \mathrm{v}$ & $17 v$ & $19 r$ & $23 r$ & $26 v$ & $27 r$ \\
\hline XRF & $\times$ & $\times$ & $x$ & $\times$ & & $\times$ & & $\times$ & $x$ & $\times$ & $\times$ & $\times$ & $\times$ \\
\hline rFTIR & $\times$ & & & & & $x$ & & $x$ & & $x$ & $x$ & & \\
\hline Raman & $x$ & $x$ & $x$ & $\times$ & & $x$ & & $x$ & & & & & \\
\hline FORS & $\times$ & & & & & $x$ & & & & $x$ & & & \\
\hline XRF scanning & & & & & & $\times$ & & & & & & & \\
\hline \multirow[t]{2}{*}{ Technique } & \multicolumn{13}{|l|}{ Folio } \\
\hline & $35 r$ & $37 r$ & $37 v$ & & $44 v$ & & $47 v$ & & $48 v$ & $49 r$ & $49 v$ & $51 \mathrm{v}$ & $52 r$ \\
\hline XRF & $x$ & $x$ & $\times$ & & $x$ & & $x$ & & $x$ & $\times$ & $\times$ & $\times$ & $\times$ \\
\hline rFTIR & & $x$ & $\times$ & & & & $\times$ & & $x$ & & $x$ & & \\
\hline Raman & & $x$ & $x$ & & $x$ & & $x$ & & & & & $\times$ & \\
\hline FORS & & $x$ & & & & & $x$ & & & & & & \\
\hline XRF scanning & & & & & & & $x$ & & & & & & \\
\hline
\end{tabular}

$\mathrm{mp})$, rFTIR on 10 folios (66 mp), Raman on 11 folios (36 mp), and FORS on 5 folios (19 mp). XRF scanning was performed on folios $10 \mathrm{v}$ and $47 \mathrm{v}$.

Images of folios $1 \mathrm{r}, 10 \mathrm{v}, 19 \mathrm{r}, 37 \mathrm{r}$, and $47 \mathrm{v}$ which also show the analyzed points are provided in Additional files $1,2,3,4$ and 5 .

\section{XRF single point measurements}

The manuscript was examined with a spectrometer Type ELIO (XGLab S.R.L./Bruker Nano Analytics, Milano, Italy). It consists of a 4 Watt $\mathrm{X}$-ray rhodium ( $\mathrm{Rh}$ ) tube and a silicon drift chamber detector (SDD) with a resolution of $155 \mathrm{eV}$ and an active area of $25 \mathrm{~mm}^{2}$. The measuring 
geometry is $0^{\circ} / 63.5^{\circ}$ with an X-ray spot of $1 \mathrm{~mm}$ diameter. The excitation voltage was set to $40 \mathrm{kV}$ with a tube current of $60 \mu \mathrm{A}$, and the acquisition time was $60 \mathrm{~s}$. The system was operated with software version 1.6.0.29, which was also used for the evaluation of the data.

\section{XRF scanning}

Two historiated initials were scanned by using the ELIO spectrometer in steps of $0.5 \mathrm{~mm}$ in $\mathrm{x}$ and $\mathrm{y}$ direction and an acquisition time of $2 \mathrm{~s}$ per measurement point (mp). The excitation voltage was set to $40 \mathrm{kV}$ with a tube current of $100 \mu \mathrm{A}$. Two elemental maps were captured on folios $10 \mathrm{v}$ and $47 \mathrm{v}$, which consisted of $40 \times 60 \mathrm{mps}$ (scanning time $80 \mathrm{~min}$ ) and $85 \times 85 \mathrm{mps}$ (scanning time $240 \mathrm{~min}$ ). The software includes a mapping application, which allows a manual definition of the $\mathrm{keV}$ range for the elements to be mapped.

\section{rFTIR}

A spectrometer Alpha (Bruker Optics, Ettlingen, Germany) equipped with a DTGS detector and a module for external reflection was available for the rFTIR measurements. An image of the IR spot obtained by an Osiris camera for infrared reflectography (Opus Instruments, Norwich, UK) showed that it covers $\sim 5 \mathrm{~mm}$ (roughly circular), with increasing intensity towards the center. However, Legrand et al. reported an experimentally determined effective spot size of $1.8 \mathrm{~mm}$ (horizontal) and $2.1 \mathrm{~mm}$ (vertical) [13]. The background was acquired using a gold mirror and reflectance spectra (average of either 64 or 128 scans) were collected in the range between 7000 and $375 \mathrm{~cm}^{-1}$ with a resolution of $4 \mathrm{~cm}^{-1}$. The operation of the instrument and the evaluation of the spectra was performed with Opus 7.5 software. The reflectance spectra were evaluated either without processing, or occasionally after calculation of absorption index spectra by Kramers-Kronig transformation in the range between 4000 and $375 \mathrm{~cm}^{-1}$, followed by baseline correction (concave rubberband correction, 10 iterations, 64 baseline points). The available spectral databases included reflectance spectra of materials in the material collection of the Institute of Science and Technology in Art, Academy of Fine Arts Vienna, as well as spectra measured in transmission mode from the IRUG 2007 database [14].

\section{Raman spectroscopy}

Raman analyses were performed with a micro-Raman spectrometer Pro-Raman-L-Dual-G (Enwave Optronics, Irvine, CA, USA) equipped with a $785 \mathrm{~nm}$ Diode Laser $(\sim 350 \mathrm{~mW})$, a fiber optic probe with $50 \times$ long working distance objective (Leica Microsystems, Wetzlar, Germany), and a Peltier cooled two dimensional CCD array detector. The spot size was $50 \mu \mathrm{m}$ and spectra were collected in the range between 3000 and $100 \mathrm{~cm}^{-1}$ with a resolution of $8 \mathrm{~cm}^{-1}$. RamanReader data collection software was applied to operate the system and the spectra obtained were compared to reference spectra from the collection of samples by using Opus 7.5.

\section{Fiber optic reflectance spectroscopy (FORS)}

FORS analyses were conducted using a spectrometer MSP400 (J\&M Analytik AG, Aalen, Germany) equipped with a halogen lamp, a 244 diode array detector, and quartz fiber optics. The self-built probe [15] has a $0^{\circ} / 45^{\circ}$ measuring geometry and a collimator enables a spot diameter of $1.5 \mathrm{~mm}$ (see Additional file 6). Reflectance spectra were collected either in the range between 380 and $900 \mathrm{~nm}$ or 320 and $1147 \mathrm{~nm}$. The spectrometer was operated utilizing TidasDAQ3 software. Additionally, Panorama 4.0 software was used for spectra evaluation by comparison to a database with spectra obtained in materials from the collection of samples mentioned above.

\section{Results and discussion}

\section{Materials used to make the Vienna Moamin}

A summary of the basic materials used by the scribes and illuminators for the Vienna Moamin including the results of the respective analytical techniques is given in Table 2 and the results are discussed more in detail subsequently.

In general, our results suggest that the color palette includes colorants which have been widely used for book decoration throughout the Middle Ages, e.g. lead white, azurite, minium or vermilion. On the other hand, materials such as mosaic gold or Brazilwood lake seem to have been used not before the late thirteenth century-the time when the Moamin was manufactured. Interestingly, Nabais et al. [16] report an almost similar color palette compared to the Moamin (except the additional use of lapis lazuli) in a Galician-Portuguese songbook (The Ajuda Songbook) dated around 1300.

\section{The parchment}

The detection of calcium carbonate $\left(\mathrm{CaCO}_{3}\right)$ by rFTIR and Raman on the parchment surface argues for the use of lime for dehairing of the skins, as residues of lime may react with atmospheric $\mathrm{CO}_{2}$ to form $\mathrm{CaCO}_{3}$ [17] and/ or whitening of the parchment with chalk. The bands with maxima at $1450 \mathrm{~cm}^{-1}$ and $875 \mathrm{~cm}^{-1}$ in the absorption index spectra match with the IRUG reference calcite MP00108, and were assigned to $v_{3}$-asymmetric $\mathrm{CO}_{3}$ stretching and $\mathrm{v}_{2}$-asymmetric $\mathrm{CO}_{3}$ deformation, respectively [18]. Additionally, calcium soaps were determined by rFTIR in a single measurement point on folio $37 \mathrm{r}$, which possibly represents a reaction product of residual subcutaneous fat from the skins with calcium carbonate. 
Table 2 Materials determined by XRF (including XRF scanning), rFTIR, Raman, and FORS in the Vienna Moamin. Minor elements determined by XRF are presented in brackets

\begin{tabular}{|c|c|c|c|c|c|}
\hline Material & Conclusion & XRF & rFTIR & Raman & FORS \\
\hline \multicolumn{6}{|c|}{ Carrier material for writing and painting } \\
\hline Whitish folios & $\begin{array}{l}\text { Parchment with } \mathrm{CaCO}_{3} \\
\text { content }\end{array}$ & $\mathrm{S}, \mathrm{Cl}, \mathrm{K}, \mathrm{Ca},(\mathrm{Fe})$ & $\begin{array}{l}\text { Parchment, calcium } \\
\text { carbonate }\end{array}$ & Calcium carbonate & Uncharacteristic \\
\hline \multicolumn{6}{|l|}{ Writing inks } \\
\hline Brown, main text & Iron gall ink & $\mathrm{Fe},(\mathrm{Cu}, \mathrm{Pb})$ & $\begin{array}{l}\text { Uncharacteristic partially } \\
\text { calcium oxalate }\end{array}$ & Iron gall ink & Increasing reflectance in NIR \\
\hline Brown, marginal notes & Iron gall ink & $\mathrm{S}, \mathrm{K}, \mathrm{Fe},(\mathrm{Cu})$ & Uncharacteristic & Iron gall ink & Not analyzed \\
\hline Red ink, main text & Vermilion & $\mathrm{S}, \mathrm{Hg}$ & Uncharacteristic & Vermilion & Vermilion \\
\hline \multicolumn{6}{|l|}{ Colorants } \\
\hline White & Lead white & $\mathrm{Pb}$ & Lead white & Not analyzed & Uncharacteristic \\
\hline Blue & Azurite & $\mathrm{Fe}, \mathrm{Cu}$ & Azurite & Not analyzed & Azurite \\
\hline Light blue & Azurite, lead white & $\mathrm{Fe}, \mathrm{Cu}, \mathrm{Pb}$ & Azurite, lead white & Not analyzed & Azurite \\
\hline Green & Orpiment, indigo, gypsum & $\mathrm{Si}, \mathrm{S}, \mathrm{K},(\mathrm{Fe})$, As & Gypsum & Orpiment \& indigo & Indigo \\
\hline Gold-beige & Mosaic gold & $\mathrm{S}, \mathrm{Sn}$ & $\begin{array}{l}\text { Uncharacteristic partially } \\
\text { gypsum }\end{array}$ & Not analyzed & Not analyzed \\
\hline Yellow & Ochre, kaolin, lead white & $\mathrm{Si}$, (Ti), $\mathrm{Fe}, \mathrm{Cu}, \mathrm{Pb}$ & Kaolin, lead white & Not analyzed & Not analyzed \\
\hline Orange & Minium & $\mathrm{Pb}$ & $\begin{array}{l}\text { Parchment bands attenu- } \\
\text { ated }\end{array}$ & Minium & Not analyzed \\
\hline Red & Vermilion & $\mathrm{Hg}, \mathrm{S}$ & Uncharacteristic & Vermilion & Vermilion \\
\hline Red & Brazilwood lake & $\mathrm{Ca}$ & Calcium carbonate & Not analyzed & Brazilwood lake \\
\hline Pink & $\begin{array}{l}\text { Brazilwood lake, lead } \\
\text { white }\end{array}$ & $\mathrm{Ca}, \mathrm{Pb}$ & $\begin{array}{l}\text { Calcium carbonate, lead } \\
\text { white }\end{array}$ & Not analyzed & Brazilwood lake \\
\hline Brown & Iron oxide & $\mathrm{Ca}, \mathrm{Fe}$ & Uncharacteristic & Iron oxide & Not analyzed \\
\hline Brown & Iron oxide with silicate & $\mathrm{Si}, \mathrm{S}, \mathrm{Ti}, \mathrm{Fe}$ & Not analyzed & Not analyzed & Not analyzed \\
\hline Black & Carbon black & $\begin{array}{l}\text { Only elements } \\
\text { from parch- } \\
\text { ment }\end{array}$ & Uncharacteristic & Carbon black & Carbon black \\
\hline
\end{tabular}

The main fatty acids in animal fat are oleic acid, palmitic acid, and stearic acid [19], which all show relatively similar FTIR spectra [20]. The characteristic bands of calcium stearate are observed at $2957 \mathrm{~cm}^{-1}$ (antisymmetric $v_{\mathrm{a}} \mathrm{CH}_{3}$ ), 2922 and $2852 \mathrm{~cm}^{-1}$ (antisymmetric $v_{\mathrm{a}} \mathrm{CH}_{2}$ and symmetric $v_{\mathrm{s}} \mathrm{CH}_{2}$ ), as well as 1580 and $1546 \mathrm{~cm}^{-1}$ (antisymmetric $v_{\mathrm{a}} \mathrm{COO}^{-}$stretching in unidentate and bidentate coordination with calcium ions) [21]. Calcium soaps were also determined in various other manuscripts during our investigations [6], e.g. in Codex slavicus 8 (fourteenth century, Austrian National Library), but the non-invasive techniques used did not allow to receive further information concerning the origin of the material.

\section{Brown and red writing inks}

XRF analyses revealed different elemental compositions of the brown inks used for the main body of the text (Fe with low $\mathrm{Cu}$ and $\mathrm{Pb}$ contents) and the marginal notes ( $\mathrm{S}, \mathrm{K}$ and $\mathrm{Fe}$ with low $\mathrm{Cu}$ content). In both cases the Raman spectra showed the characteristic spectral features of iron gall inks, which were, according to the literature [22-24], at about 1576, 1478 (strong),
1329 (strong), 535, and $373 \mathrm{~cm}^{-1}$ (Fig. 3a). FORS spectra of the brown inks of the main text showed increasing reflectance values towards the NIR region (Fig. 3b) analogously to the iron gall ink spectra reported by Aceto and Cala [25].

No indications of iron gall inks were obtained by rFTIR. The relatively large spot size of the rFTIR instrument did not allow an analysis of the ink alone without the surrounding parchment, which contributed to the spectra obtained. However, a comparison of several measurement points in the main text to the blank parchment showed increased intensities in the absorption index spectra in the regions of the amide $\mathrm{I}(\mathrm{vCO}, \mathrm{vCN}$ and $\delta \mathrm{NH}$ ) and the $\omega \mathrm{CH}_{2} / \delta \mathrm{CH}$ (methine) parchment bands at $1645 \mathrm{~cm}^{-1}$ and $1334 \mathrm{~cm}^{-1}$ [26]. These features overlap with the antisymmetric $v_{\mathrm{a}} \mathrm{CO}$ and symmetric $v_{\mathrm{s}} \mathrm{CO}$ vibrations of calcium oxalate dihydrate (weddellite) [27] and match with the IRUG reference spectrum weddellite MP00425. The formation of oxalates in iron gall inks indicates oxidative degradation of organic compounds [28], although the mechanisms are currently not well understood. 

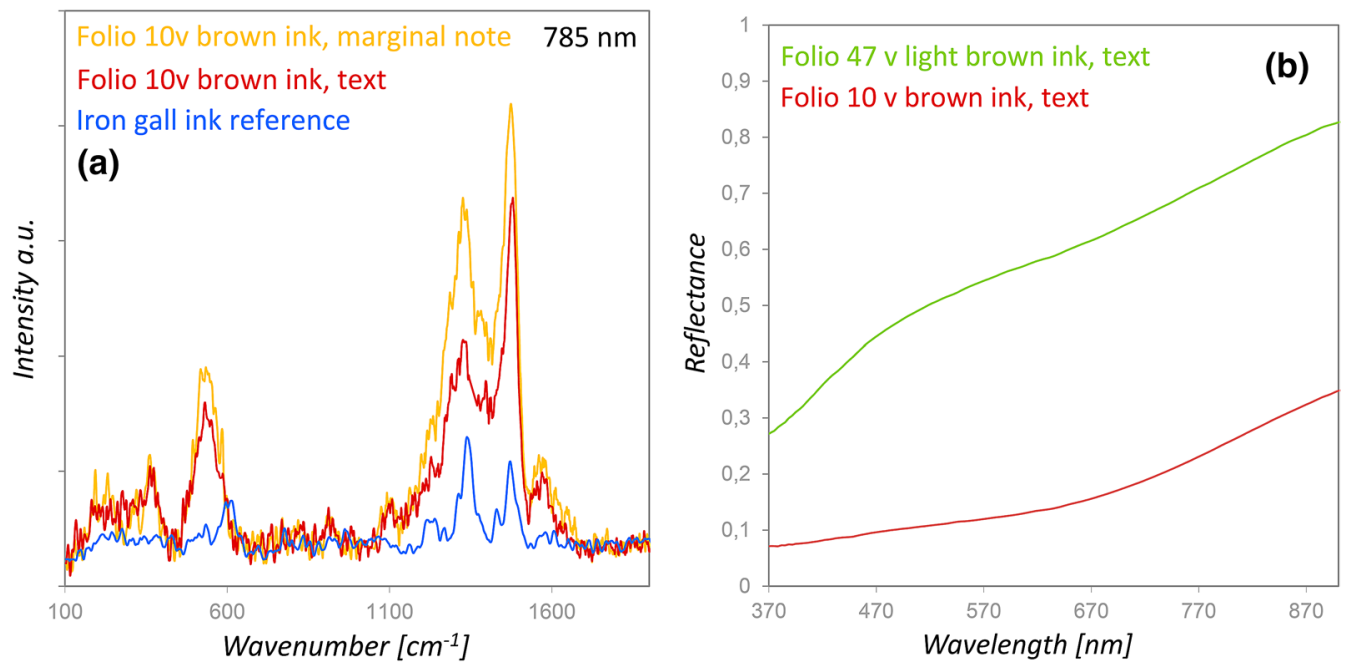

Fig. 3 a Comparison of Raman spectra of the brown ink in the text (red) and the marginal note (yellow) on folio 10v with an iron gall ink reference spectrum (blue) and $\mathbf{b}$ a FORS spectrum of the brown ink in the text of folio $1 \mathrm{v}$ compared with the light brown ink (green) on folio $47 \mathrm{v}$

Vermilion was identified in the red writing inks, which was proved by XRF, Raman and FORS. Characteristic for semiconductor pigments, the FORS spectra of vermilion showed a sharp S-shaped increase of reflectance starting from ca. $550 \mathrm{~nm}$ and an inflection point at ca. $600 \mathrm{~nm}$. The characteristic elements were $\mathrm{Hg}$ and $\mathrm{S}$, although the $\mathrm{XRF} \mathrm{K \alpha}$ line of $\mathrm{S}(2.305 \mathrm{keV})$ is coinciding with $\mathrm{Pb} \mathrm{M} \alpha-$ $(2.346 \mathrm{keV})$ and $\mathrm{Hg} \mathrm{M} \alpha$-lines $(2.195 \mathrm{keV})$ in two spectra from folios $3 \mathrm{r}$ and $37 \mathrm{v}$. However, these two measurement points were not analyzed by complementary methods and it remains unclear, whether the $\mathrm{Pb}$ contents resulted from an addition of minium to the inks or from a material transfer from surrounding areas containing minium or lead white. The spectra obtained by rFTIR were similar to the spectra measured on the bare parchment, as vermilion only absorbs at wavenumbers below the range available with the spectrometer used $\left(375 \mathrm{~cm}^{-1}\right)$ [29].

\section{Blue}

The identification of azurite $\left(\mathrm{Cu}_{3}\left(\mathrm{CO}_{3}\right)_{2}(\mathrm{OH})_{2}\right)$ in the Vienna Moamin has already been published in an rFTIR study discussing the characteristic spectral features of the pigment mixed with various binding media [30]. XRF detection of $\mathrm{Cu}$ supported the rFTIR results. In addition, smaller quantities of $\mathrm{Fe}$ and $\mathrm{Si}$ indicate typical impurities of mineral azurite, particularly hematite and silicates [31], although no evidence for silicates could be derived from the rFTIR spectra. Additionally, $\mathrm{Pb}$ was detected by XRF in most of the measurement points in the blue areas, which mainly derives from fine white decorative lines over the blue ground layer (Fig. 1). Various reflectance maxima were observed

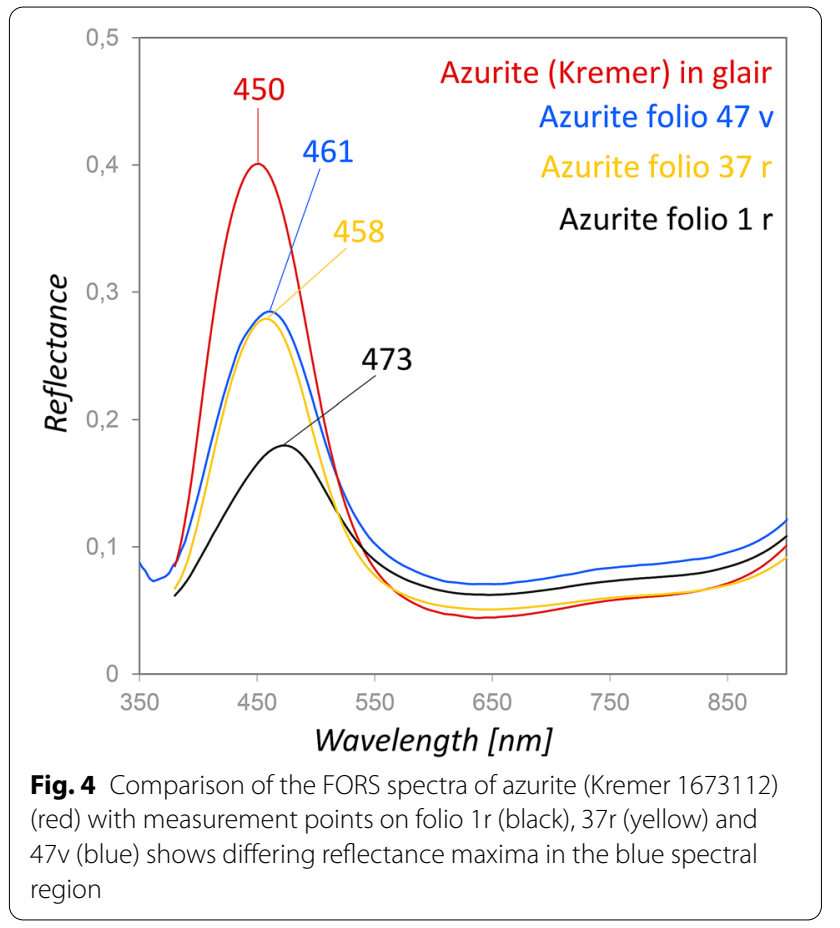

in the FORS spectra (Fig. 4), particularly $473 \mathrm{~nm}$ on folio $1 \mathrm{r}, 458 \mathrm{~nm}$ on folio $37 \mathrm{r}$, and $461 \mathrm{~nm}$ on folio $47 \mathrm{v}$, compared to $450 \mathrm{~nm}$ in case of a contemporary natural azurite pigment (Kremer, order number: 1673112). The blue on folio 1r appears greenish and dull in comparison to the other examples due to the lower reflectance at the maximum as well as its shift to higher wavelengths. 
A mixture of azurite and lead white could be determined in the light blue areas, as detected on folio $37 \mathrm{r}$ by XRF $(\mathrm{Cu}, \mathrm{Pb})$ and rFTIR. The bands in the fingerprint region of the absorption index spectra match with the IRUG references azurite MP00001 and lead white MP00107 (see Additional file 7). Furthermore, two characteristic bands at $4378 \mathrm{~cm}^{-1}$ (overtone $3 v_{3}$ ) and $4244 \mathrm{~cm}^{-1}$ (combination $v+\delta \mathrm{O}-\mathrm{H}$ ) in the rFTIR spectrum enable an easy identification of azurite [30]. The $v_{3} \mathrm{CO}_{3}^{2-}$ asymmetric stretching vibration of azurite at around $1400 \mathrm{~cm}^{-1}$ overlaps with the analogous feature of lead white. However, lead white was identified preferably in the absorption index spectrum by means of the sharp $v_{4} \mathrm{CO}_{3}$ in-plane bending vibration at $683 \mathrm{~cm}^{-1}$ and the weaker $v_{1} \mathrm{CO}_{3}$ symmetric stretching vibration at $1045 \mathrm{~cm}^{-1}$ [32].

\section{Green}

Green areas in the historiated initials and decorations were painted by using a mixture of indigo and orpiment. Orpiment was detected by XRF (As, S) and Raman spectroscopy, whereas the characteristic spectral features of indigo matched with the respective Raman and FORS reference spectra (see Additional file 8). The indigo content was too low for a determination by rFTIR, but the spectra showed the characteristic features of gypsum. It has been reported [33, 34] that gypsum often is associated with orpiment derived from geothermal or volcanic environments and hence, it can be considered most probably as an impurity of the yellow pigment. The characteristic bands of gypsum were inverted in the rFTIR spectra showing maxima at ca. 1146,675 , and $601 \mathrm{~cm}^{-1}$, corresponding to the $v_{3} \mathrm{SO}_{4}$ and $v_{4} \mathrm{SO}_{4}$ vibrations [35]. The inversion of bands is often observed in reflection FTIR spectra of oxyanion pigments and results from the specularly reflected proportion of the radiation [36]. Moreover, combination bands of $v_{1}$ and $v_{3}$ modes of $\mathrm{SO}_{4}$ at 2227 and $2120 \mathrm{~cm}^{-1}$ appear similar to transmission spectra, e.g. IRUG reference gypsum MP00105, and the $\mathrm{OH}$ bands were generally almost invisible in the reflectance spectra for unknown reasons (Fig. 5).

\section{Yellow}

The elements $\mathrm{Si}, \mathrm{Ti}, \mathrm{Fe}$, and $\mathrm{Pb}$ were detected in a yellow color solely on folio 1r by means of XRF, which suggests

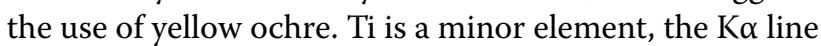
is very weak and the $K \beta$ line is imperceptible. The content of $\mathrm{Ti}$ and other minor elements $(\mathrm{K}, \mathrm{Ca})$ is characteristic for individual ochres and is used for their classification $[37,38]$. However, we did not quantify the Ti content and $\mathrm{K}$ and $\mathrm{Ca}$ could not be determined due to the relatively high contents of both elements in the parchment. The rFTIR results as well as the image from the on-board

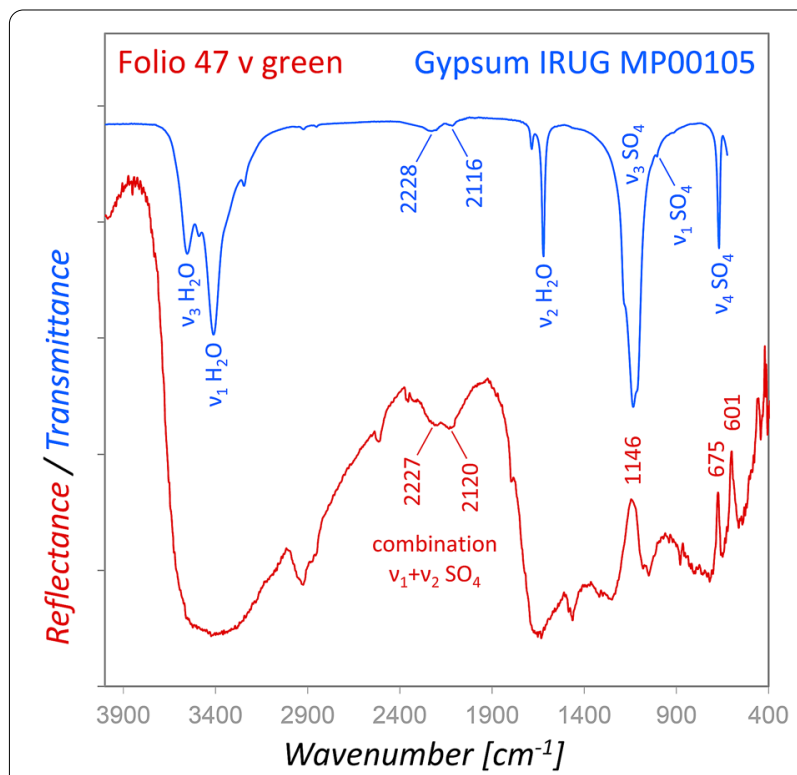

Fig. 5 Comparison of the reflectance spectrum obtained from a green area (red) with a reference spectrum of gypsum measured in transmission (blue). The strong $\mathrm{SO}_{4}$ vibrations $\left(\mathrm{v}_{3}\right.$ and $\left.\mathrm{v}_{4}\right)$ are inverted, but the $\mathrm{OH}$ bands are almost invisible

camera suggested that $\mathrm{Pb}$ derives from fine decoration lines painted with lead white, which have partially flaked off. Silicon is a main constituent of kaolinite. Si-O stretching bands (between 1130 and $970 \mathrm{~cm}^{-1}$ ) as well as $\mathrm{Al}-\mathrm{O}-\mathrm{Si}$ deformation $\left(537 \mathrm{~cm}^{-1}\right)$ and $\mathrm{Si}-\mathrm{O}-\mathrm{Si}$ deformation $\left(467 \mathrm{~cm}^{-1}\right)$ bands [39] are inverted in the reflectance spectrum. Furthermore, $\mathrm{OH}$ stretching bands in the corresponding absorption index spectrum at 3690, 3669 and $3617 \mathrm{~cm}^{-1}$ match with the IRUG kaolinite reference MP00109.

\section{Gold-beige}

The color was often applied in the historiated initials, as well as ornamental decorations. It appears moderately shiny. In three single measurement points, Sn and $\mathrm{Pb}$ were detected by XRF, although in various ratios. This would, in principle, argue for the use of lead tin yellow $\left(\mathrm{Pb}_{2} \mathrm{SnO}_{4}\right)$. In fact, an in-depth evaluation and interpretation of the XRF scanning data, which will be discussed below in the respective section, showed that mosaic gold $\left(\mathrm{SnS}_{2}\right)$ was used in this manuscript. The rFTIR results indirectly supported this finding, as no characteristic spectral features could be observed in the region between ca. 580 and $400 \mathrm{~cm}^{-1}$, where Nodari and Ricciardi [40] reported inverted bands in the rFTIR spectra of lead tin yellow type 1 in egg white at 569, 504,459 , and $427 \mathrm{~cm}^{-1}$. In the rFTIR spectra the parchment bands were strongly attenuated by the pigment layer and mainly very broad spectral features were 
observable. In addition, the spectrum obtained on folio $37 \mathrm{r}$ showed the characteristic vibrations of gypsum $v_{3}$ $\mathrm{SO}_{4}$ at 1141 and $1119 \mathrm{~cm}^{-1}$, as well as $\mathrm{v}_{4} \mathrm{SO}_{4}$ at 669 and $594 \mathrm{~cm}^{-1}$, though relatively weak. According to the literature, mosaic gold, also known as aurum musicum or purpurinus, is a golden-brown pigment with a very subdued sparkle, which was first used in the thirteenth century, but still was considered a novelty by the treatise writers in the fourteenth century $[41,42]$. In this respect, the detection of mosaic gold in the Moamin is an example of a quite early use of mosaic gold. Nabais et al. [16] also consider the detection of mosaic gold in the Galician-Portuguese Ajuva Songbook dated around 1300 as an early occurrence in manuscripts. Unfortunately, the gold-beige color was not analyzed by Raman spectroscopy or FORS, which also would be appropriate methods for the identification of the pigment. The reason therefore is that the Raman analyses mainly focused on the characterization of the writing inks, black and green pigments and FORS mainly on the blue, green and red pigments. We experienced in previous analyses of various manuscripts that particularly inks, as well as black and green pigments can hardly be characterized by XRF, rFTIR or FORS. Furthermore, the limited access to the manuscript did not allow a complete study of the materials by all methods applied. However, the detection of the pigment in manuscripts has been reported by means of a single characteristic Raman band at $314 \mathrm{~cm}^{-1}$ [43]. This band can be assigned to a phonon mode of the $2 \mathrm{H}$ polytype of $\mathrm{SnS}_{2}$ [44], and very similar results were obtained by excitation with either $514 \mathrm{~nm}$ or $785 \mathrm{~nm}$ lasers [45]. Moreover, a characteristic FORS spectrum with inflection points at 500 and $525-535 \mathrm{~nm}$ has been published by Aceto et al. [46]. It should be mentioned that the unique optical characteristics of mosaic gold will surely lead us to use Raman spectroscopy and FORS in addition to XRF for a comprehensive identification in future investigations.

\section{Orange}

Minium $\left(\mathrm{Pb}_{3} \mathrm{O}_{4}\right)$ was used frequently in initials and decorations. The XRF analysis showed that in orange areas $\mathrm{Pb}$ often was detected together with $\mathrm{Cu}$, which most probably derives from a transfer of material from blue areas on the folios with azurite. Minium was also identified by Raman spectroscopy. Three bands in the rFTIR absorption index spectra at 531, 531, and $460 \mathrm{~cm}^{-1}$ are in accordance with the data reported for minium measured by using ATR-FTIR [29]. Although the pigment does not absorb in the region between 1700 and $1100 \mathrm{~cm}^{-1}$, the bands of parchment are strongly attenuated compared to the spectra of the blank parchment.

\section{Red and pink}

High amounts of Ca were detected by XRF in the dark red color mainly used in the historiated initials, along with $\mathrm{K}$ and few $\mathrm{Cu}$. In accordance with these results the rFTIR analyses showed high quantities of $\mathrm{CaCO}_{3}$. However, an identification of the colorant was not possible by these methods. In contrast, FORS analyses indicated the presence of Brazilwood lake, which was further used in

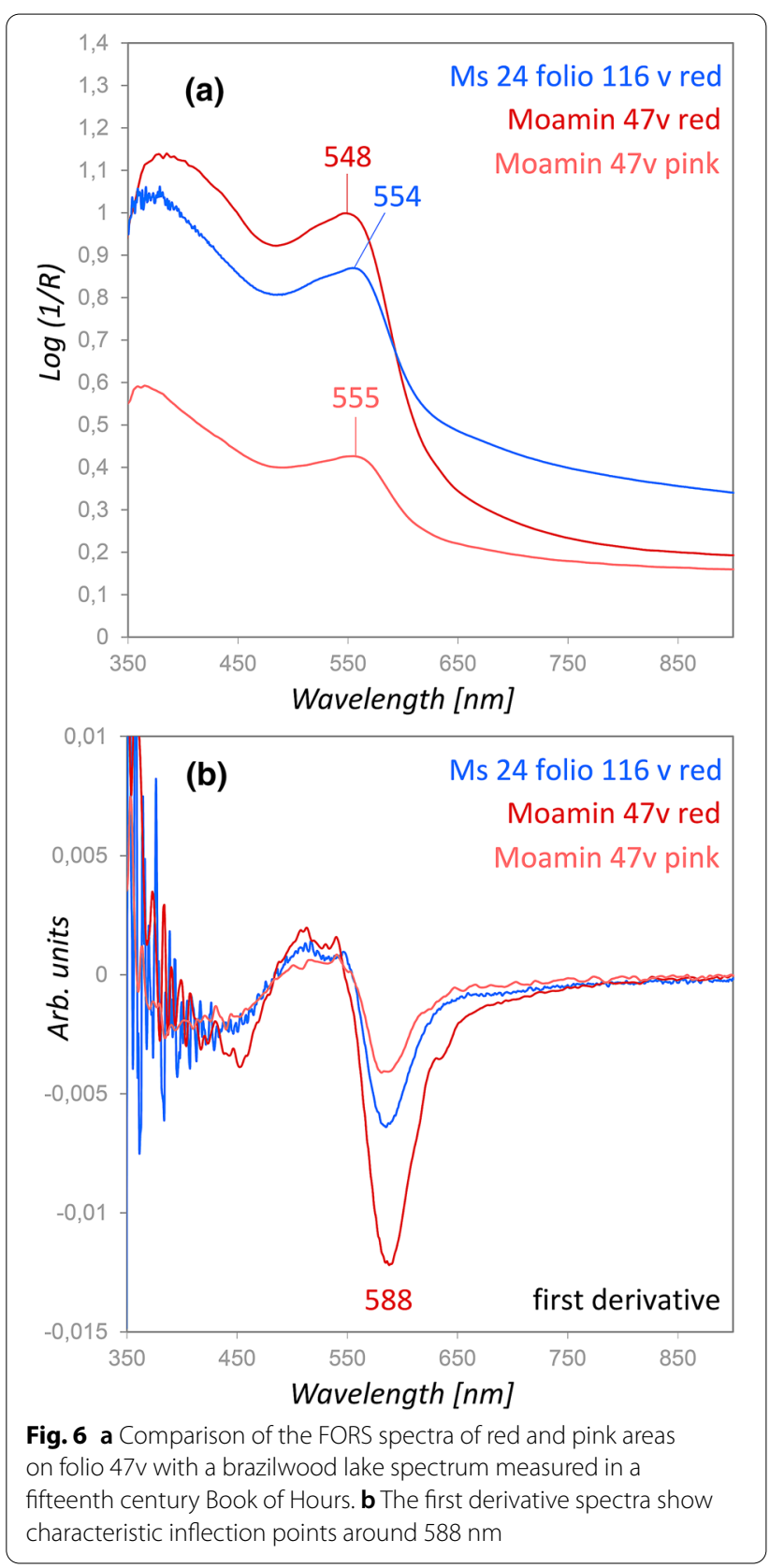


combination with lead white for pink hues in the initials. It has been reported that Brazilwood-based colors were used from the fourteenth century [16] and hence, its presence in the Moamin would be an example of an early use. The spectra from a red and a pink area on the middle initial in Fig. 1 (folio 47v) are depicted in Fig. 6a) and show the characteristic spectral features of the Brazilwood chromophore as published by Melo et al. [43] and Aceto et al. [46]. Moreover, a Brazilwood lake spectrum from a fifteenth century Book of Hours (ms 24 in the library of Palalacio Nacional de Mafra (PNM) in Portugal) matches well with the spectra obtained in the Moamin. The spectrum was kindly provided by Tatiana Vitorino, who is coauthor of the paper mentioned before. The first derivative of the spectra is shown in Fig. 6b). The inflection points are very close to each other around $588 \mathrm{~nm}$, which further underlines the similarity.

With respect to the identification of $\mathrm{CaCO}_{3}$ by rFTIR, Melo et al. [43] reported that $\mathrm{CaCO}_{3}$ was frequently added "either to help pigment precipitation or to contribute to the substrate". The red color was not analyzed by using Raman spectroscopy. However, to our knowledge an identification of Brazilwood lakes in manuscripts by using a $785 \mathrm{~nm}$ laser has not been reported so far and even Brazilwood extracts without binding medium seem to produce strong fluorescence with only very weak Raman peaks [47]. Moreover, Mulholland et al. [48] tested various excitation sources $(488,532,633,785,830$, and $1064 \mathrm{~nm}$ ) for the analysis of a Brazilwood watercolor pigment and observed strong fluorescence for any excitation source, and peaks were only observed in case of the $1064 \mathrm{~nm}$ laser despite of the high level of fluorescence. This demonstrates the limitations of Raman spectroscopy when only one or two excitation lasers are available.

\section{Brown}

Brown iron oxide was detected on folio $1 \mathrm{r}$ by XRF (Ca and $\mathrm{Fe}$ ) and Raman spectroscopy (see Additional file 9). Moreover, rFTIR revealed an addition of gypsum. In contrast, brown earth pigments with contents of $\mathrm{Si}$ and $\mathrm{Fe}$ could be determined on other folios.

\section{Black}

In general, the historiated initials were outlined in black and the presence of carbon black was revealed by two broad Raman bands around 1589 and $1324 \mathrm{~cm}^{-1}$ in accordance with the literature [49], Additional file 10 shows the respective spectrum. In addition, FORS spectra showed high absorption values in the entire spectral range analyzed. Carbon black was further detected in the depiction of a dog next to the historiated initial on folio $44 \mathrm{v}$ (not shown).

\section{Binding media for illumination}

The most important binding media for illumination in medieval times were glair and Arabic gum [50, 51]. In principle, rFTIR would allow the characterization of both materials. In practice the spectra obtained from the paint layers in the illuminations generally showed a contribution of parchment bands, which may overlap with the bands of glair, as both are proteinaceous materials. Hence, no clear statements can be made. Furthermore, we could not detect spectral features of Arabic gum in the colors of the Moamin. A previous study investigating azurite in mixture with glair and Arabic gum suggested that the sensitivity of the used instrument only hardly allows the characterization of binding media [30], which might also account for other pigments.

\section{Leaf gilding technique}

Delamination of gold leaf was recognizable on several historiated initials, which enabled a direct analysis of the pink ground layer for the gold leaf on folios 1r, 16v, $19 \mathrm{r}$, and 26v. The rFTIR results showed that calcium carbonate (chalk) was used for the ground layer of folio $1 \mathrm{r}$, whereas gypsum was used on the other analyzed folios (Fig. 7). It is rather probable that the amide I and amide II bands (ca. 1655 and $1555 \mathrm{~cm}^{-1}$ ) in the rFTIR spectra obtained from folio $16 \mathrm{v}$ derive from a proteinaceous binding medium than from the parchment below, as the grounds for gilding were applied as relatively thick layers, In accordance with the rFTIR results, solely Ca was

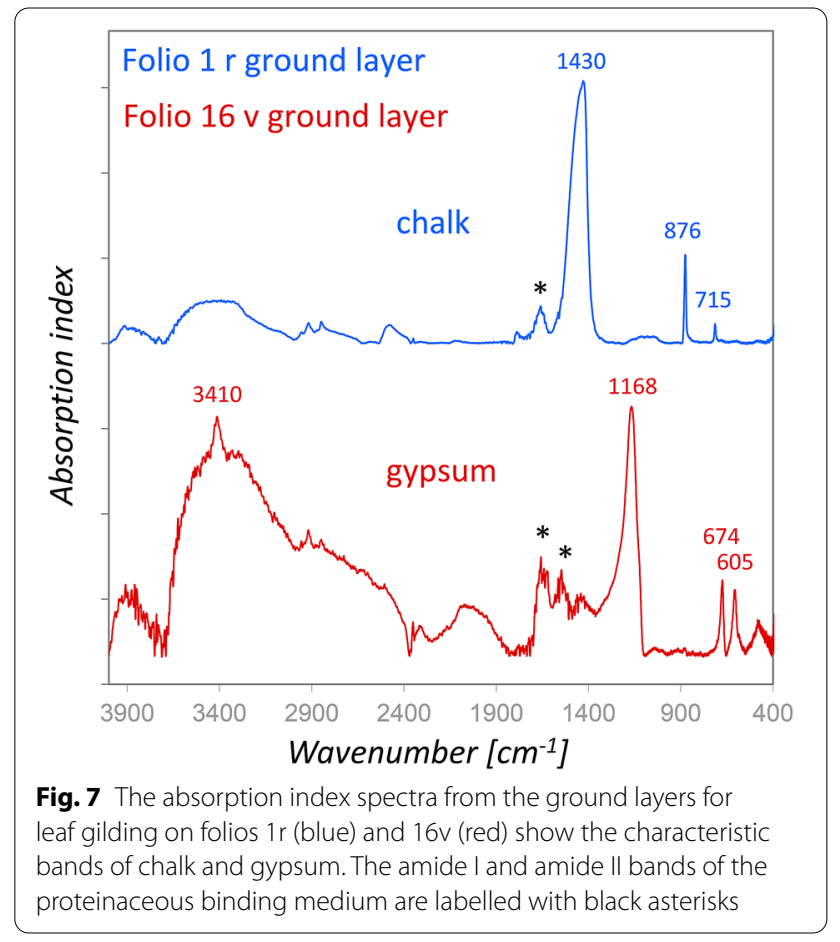


detected by XRF on folio $1 \mathrm{r}$, whereas $\mathrm{Ca}$ and $\mathrm{S}$ were detected on folios 19r and 26v. Sulfur was not unambiguously determined on folio $16 \mathrm{v}$ due to a relatively high lead content and hence an overlap of $\mathrm{Pb} \mathrm{M} \alpha$ with $\mathrm{S} \mathrm{K \alpha}$.

Furthermore, small intensities $\mathrm{Hg}$ and $\mathrm{Pb}$ were detected by XRF, indicating that the pink color consists of a mixture of vermilion and $\mathrm{a} \mathrm{Pb}$ component-minium or lead white-with gypsum. Only inconclusive results

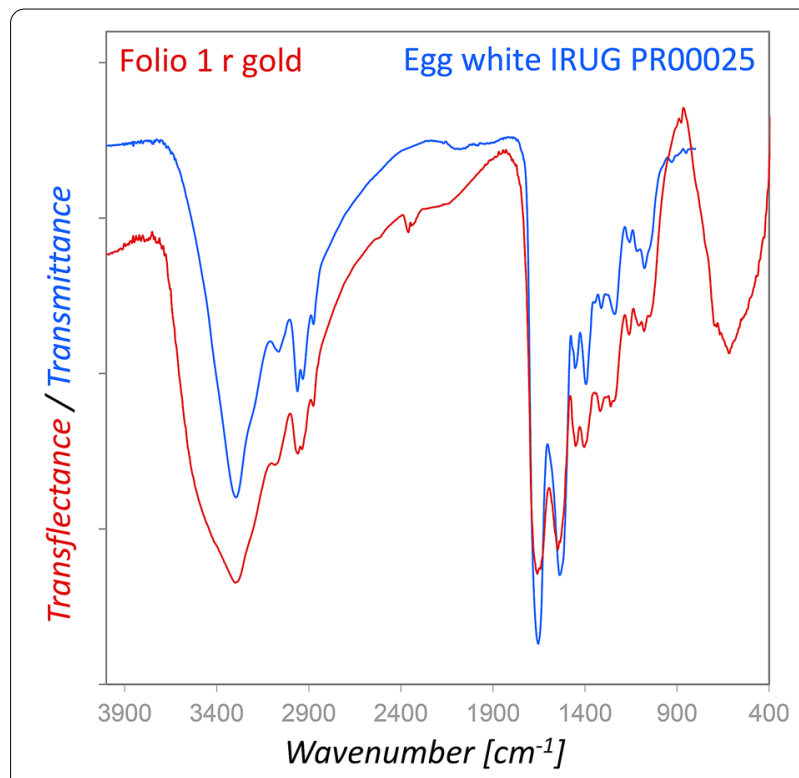

Fig. 8 Comparison of the transflectance spectrum obtained from a proteinaceous layer over the gold leaf (red) with the reference spectrum egg white IRUG PR00025 (blue, measured in transmission) were obtained by FORS regarding the colorant. However, the XFR scans on folios $10 \mathrm{v}$ and $47 \mathrm{v}$ rather suggested that mainly vermilion is present.

It was interesting to find a proteinaceous layer over the gold leaf on folio $1 \mathrm{r}$ and $47 \mathrm{v}$ by rFTIR. The gold leaf acted as a mirror for the incident infrared radiation, and hence a transflectance spectrum [52] was obtained. Figure 8 depicts that the spectrum matched well with the IRUG reference spectrum for egg white PR00025, and a comparison with other protein reference spectra showed that glues have much higher amide I/amide II ratios. However, an unambiguous identification of proteinaceous materials by FTIR is not possible.

The proteinaceous compound was further detected on the black outline which partially was applied on top of the gold. It is quite obvious that the results of these single point measurements do not allow statements about the distribution of the material and thus it remains unclear, whether the material represents a layer which was applied onto the gold by intention of the illuminator, an attempt for a consolidation of the badly preserved gilding, or simply a contamination. However, an rFTIR scanning device, as implemented by Legrand et al. [13] would have been useful in order to obtain information regarding these questions.

\section{The distinct characteristics of folio 1}

It is easily recognizable in Fig. 9 that the historiated initial on folio $1 \mathrm{r}$ remarkably differs from most of the other analyzed folios in respect of the size and the depiction of persons and birds. Further examples are provided as Additional files 2, 3, 4 and 5. The same accounts for the

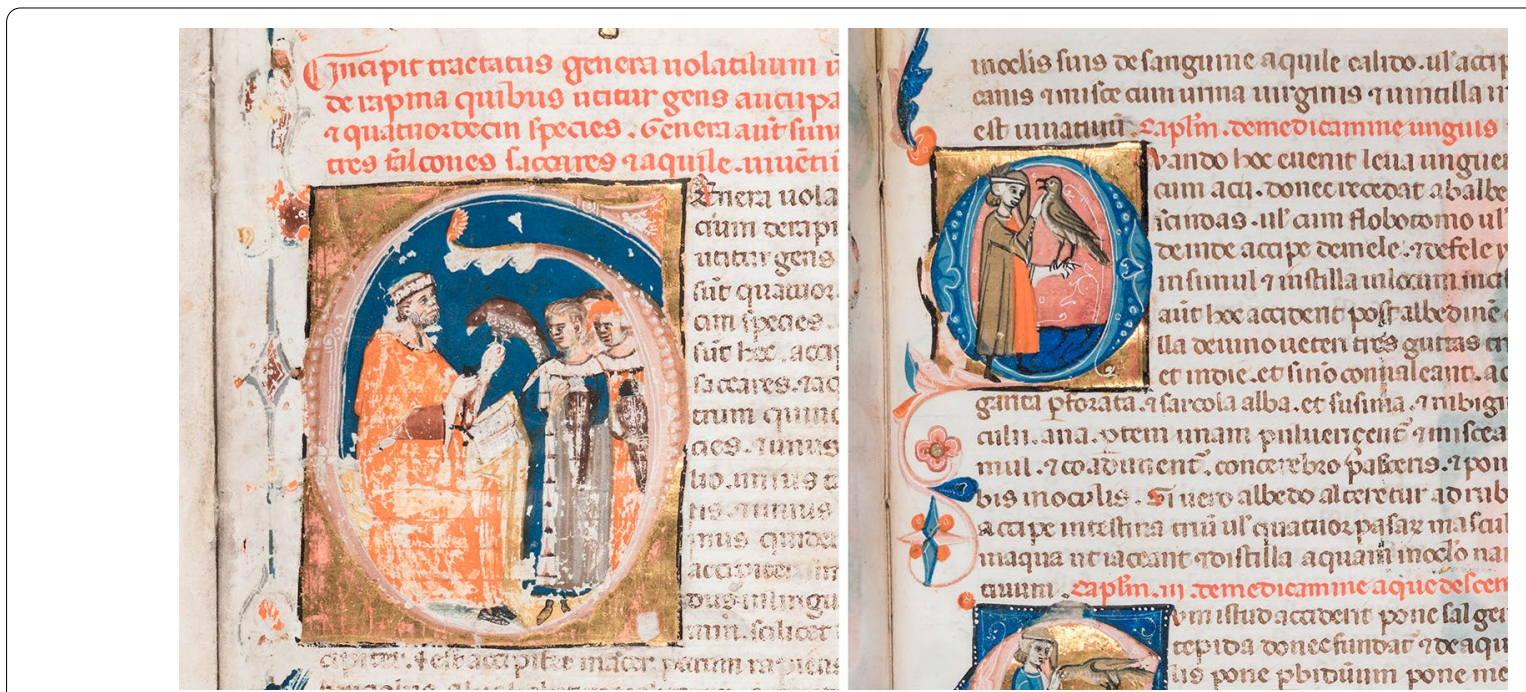

Fig. 9 The style of the historiated initial on folio $1 \mathrm{r}$ as well as its size differs remarkably from the initials on other analyzed folios such as folio $37 \mathrm{r}$, which is shown as a representative example 
artistic design of the other decorations and hence, a different authorship might be assumed. The results of the material analyses clearly supported this assumption. The most remarkable differences include a differing support for leaf gilding $\left(\mathrm{CaCO}_{3}\right.$ instead of $\left.\mathrm{CaSO}_{4}\right)$, the use of yellow ochre solely on folio $1 \mathrm{r}$, and contents of calcium soaps in various colors-white, blue, pink, brown, and grey. Interestingly, calcium soaps were not detected in the parchment, the writing red inks, and the yellow or orange areas. Calcium stearate, which is indicative of calcium soaps, can easily be determined by means of the antisymmetric and symmetric $\mathrm{C}-\mathrm{H}$ stretching vibrations at 2920 and $2850 \mathrm{~cm}^{-1}$ and the antisymmetric $\mathrm{COO}^{-}$ stretching vibrations at 1575 and $1540 \mathrm{~cm}^{-1}$ [21]. A spectrum from a blue color in comparison with the reference spectrum IRUG OF00108 is shown in Additional file 11. Analogously, we observed contents of calcium soaps in certain colors also in Codex slavicus 8 [30], which was manufactured in 1368 in the region Krbava (Croatia). Furthermore, Vieira et al. [53] reported the detection of calcium palmitate in a red colorant (lac dye) on parchment in a Koran dated 1198. This raises the question if soap was added by intention as an attempt to improve the applicability of the color. As noted in the previous section, this question can't be answered on the basis of single point measurements, which clearly shows a strong demand for rFTIR scanning devices.

\section{XRF scanning}

The elemental maps of the historiated initials on folios $10 \mathrm{v}$ and $47 \mathrm{v}$ yielded substantial information on the painting technique complementing the information obtained by single point measurements and visual light photographs. Both initials were found to be quite similar regarding the painting technique and materials used. Figures 10 and 11 show the results from the scan on folio $47 \mathrm{v}$, particularly the distribution maps of the elements characteristic for the applied colors, which are listed in Table 3.

Figure 10 shows that $\mathrm{Hg}$ (vermilion) is present in the red ink as well as the ground layer below the gold leaf. The low intensity of $\mathrm{Pb}$ in the areas with gold leaf indicates that mainly vermilion was used for the pink color of the ground layer for gilding. $\mathrm{Pb}$ indicates lead white in the falconers incarnate parts and in the pink colors of the initial letter (mixed with Brazilwood lake), as well as the light blue color in the decoration (mixed with azurite), and moreover, minium in the orange stripes on the clothing. The varying intensities in the $\mathrm{Cu}$ map indicates that the thickness of the paint layer is not uniform. Highest Fe intensities are found in the brown iron gall ink, the flesh tone, and also in the blue areas in combination with $\mathrm{Cu}$ (azurite). It is rather unlikely, that an iron compound was added intentionally to the blue azurite, as brilliant colors were preferred by the illuminators in the Middle Ages [54]. However, hematite $\left(\alpha-\mathrm{Fe}_{2} \mathrm{O}_{3}\right)$ and goethite $(\alpha-\mathrm{FeOOH})$ seem to be common impurities in azurite [31].

The quality of the image stitched by the Elio software is rather poor concerning the lateral resolution and color rendering. However, a higher quality stitched image with better color rendering could be obtained when Photomerge in Photoshop CS6 was employed, as depicted on the bottom right in Fig. 11. Additional file 12 shows the original size of the stitched image.

The Ca map shows highest intensities in the red part of the cloth, which was painted with a Brazilwood lake with high contents of calcium carbonate (detected by rFTIR in single point measurements). Lower intensities of $\mathrm{Ca}$ were detected in the ground layer for gold leaf, where also $\mathrm{S}$ was determined. This indicates that gypsum was used to prepare the ground layer, although a slight contribution of $\mathrm{S}$ from vermilion has to be considered, which was added to obtain the pink hue. The coincidence of Sn and S in the elemental maps indicates that the gold-beige color consists of mosaic gold $\left(\mathrm{SnS}_{2}\right)$. Concerning the $\mathrm{S}$ map it has to be considered that $\mathrm{Pb} \mathrm{M \alpha}(2.346 \mathrm{keV})$ partially coincides with the $\mathrm{S} \mathrm{K \alpha}(2.305 \mathrm{keV})$ signal and hence also areas with $\mathrm{Pb}$ contents are highlighted. As mentioned above, single point measurements rather argued for the use of lead tin yellow. However, the Pb map in Fig. 11 does not show significant intensities in the gold-beige areas, which strongly argues against the use of lead tin yellow. $\mathrm{K}$ was mainly detected in the gold-beige color of the cloths, the bird, and-in lower intensities-in areas with red Brazilwood lake. The role of $\mathrm{K}$ in the mosaic gold parts remains unclear since potassium compounds do not seem to have been used for its production according to medieval treatises [55]. Only a thin layer of paint was applied in the green landscape on the bottom right in the initial, where the $\mathrm{S}$ map shows relatively high intensities. XRF single point measurements indicated As and $S$ from orpiment in this area. However, it was not possible to visualize the distribution of As due to the overlap of As $\mathrm{K} \alpha(10.543 \mathrm{keV})$ with $\mathrm{Pb} \mathrm{L} \alpha(10.551 \mathrm{keV})$ and moreover, the short acquisition time during the scan did not yield enough counts for As $K \beta(11.7258 \mathrm{keV})$ in order to obtain a significant map. These results clearly demonstrate that the acquisition parameters may strongly influence the interpretation of XRF scanning data.

\section{The illuminators technique of folio $47 \mathrm{v}$}

The overall results including visual study from this historiated initial suggest that in a first step a ground layer for gold leaf was applied, consisting of gypsum (Ca), few amounts of vermilion $(\mathrm{Hg})$ and most probably a 


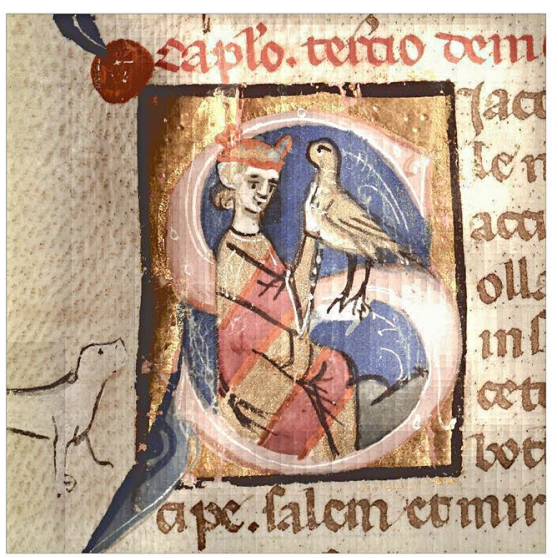

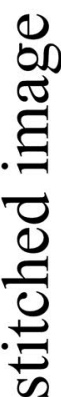
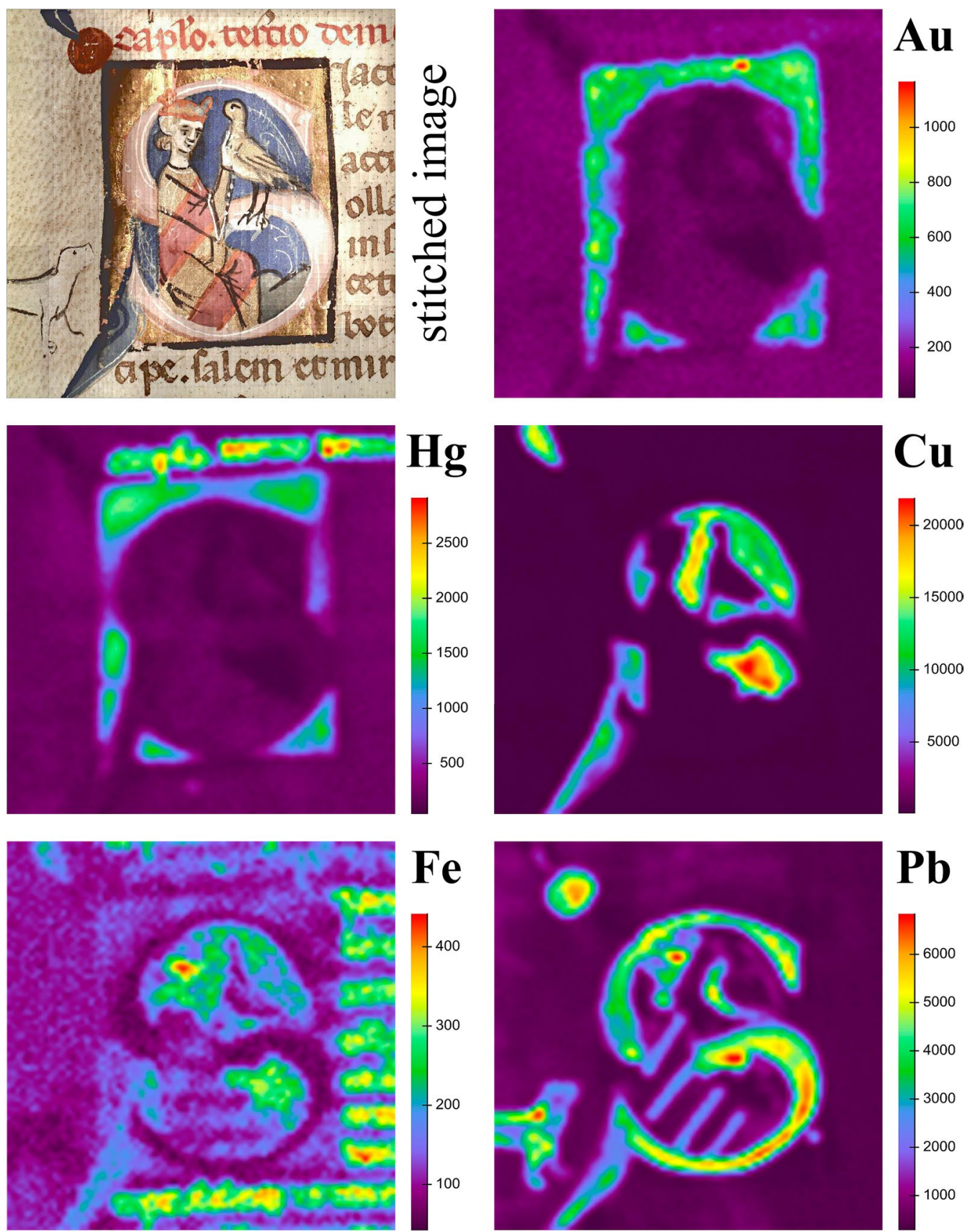

Fig. 10 Image stitched by Elio software and the elemental maps of $\mathrm{Au}, \mathrm{Hg}, \mathrm{Cu}, \mathrm{Fe}$, and $\mathrm{Pb}$ with the respective intensities (counts) obtained from the historiated initial in the middle of folio $47 \mathrm{~V}$

proteinaceous binding medium. The ground layer solely was applied on areas to be leaf gilded and defines the dimensions of the initial. After the application of gold leaf, the background colors (blue, green), the basic colors of the figural representations (gold-beige, red) and the black outlines were painted. Subsequently, the colors of the initial letters (pink), the decorations emanating from the corners (blue, light blue), and the orange stripes on the clothing were applied, which all partially cover other colors. In the last steps the figural representations were completed with black contour lines as well as white decorative lines (see Additional file 12). 

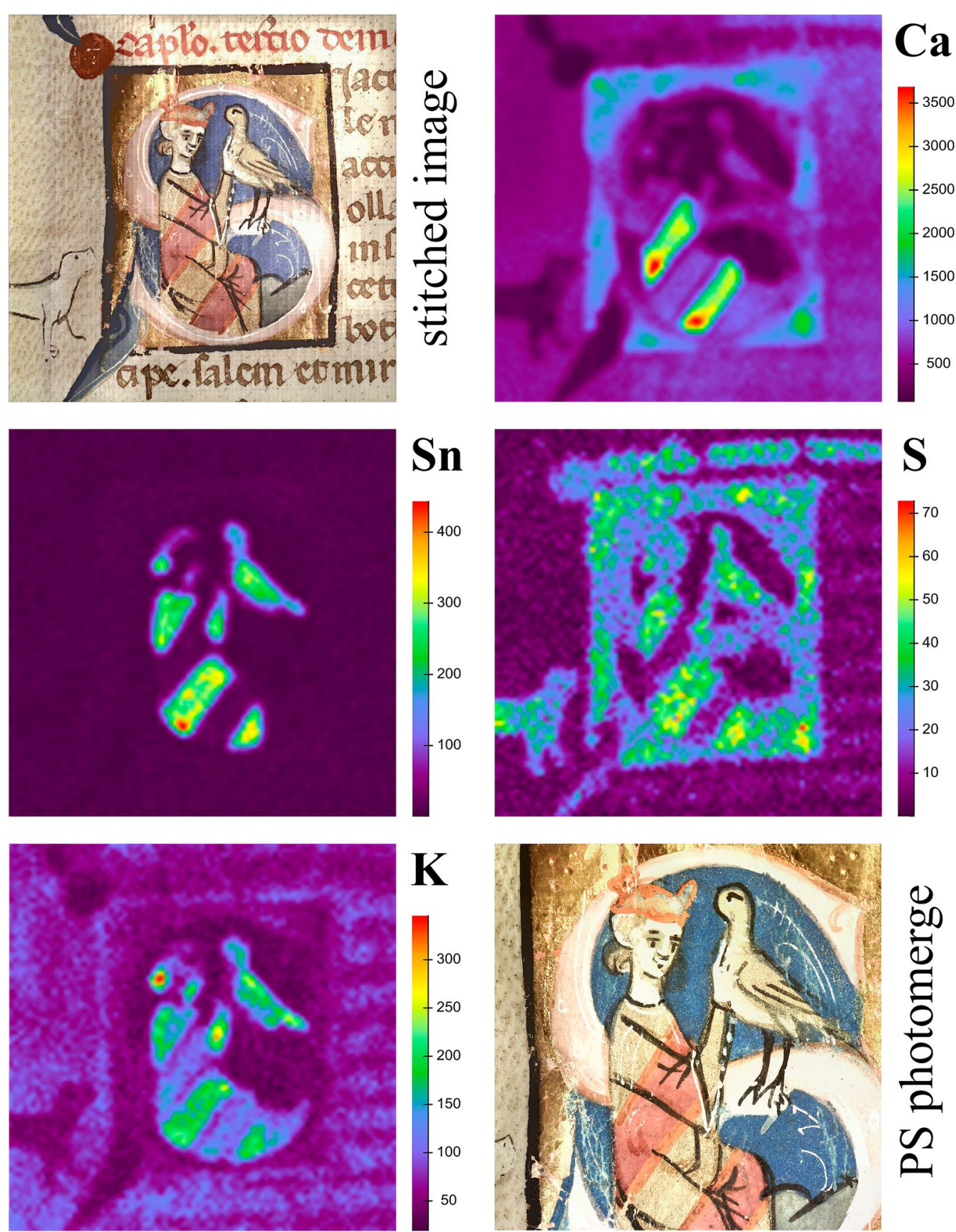

Fig. 11 Image stitched by Elio software and the elemental maps of Ca, Sn, S, and K with the respective intensities (counts) obtained from the historiated initial in the middle of folio 47v. A section of the image stitched in Photoshop CS6 is shown on the bottom right

\section{Conclusions}

The Vienna Moamin was investigated non-invasively during two measurement campaigns by using XRF, rFTIR, Raman and FORS. The first campaign aimed to characterize the materials used for this outstanding manuscript, whereas the second campaign mainly focused on the investigation of the painting technique by XRF scanning and answering questions which emerged during the evaluation of the data already obtained. The analyses allowed us to extend our knowledge about the painting techniques and the variety of materials used in the late thirteenth century in Italy. In addition, we detected materials in folio $1 \mathrm{r}$ which strongly differed from the materials in the other analyzed folios. This provides analytical 
Table 3 Characteristic elements for the colorants used on folio $47 \mathrm{~V}$

\begin{tabular}{llll}
\hline Element & X-ray emission line (keV range) & Color/material & Occurrence \\
\hline $\mathrm{S}$ & $\mathrm{Ka}(2.300-2.310)$ (partial overlap with Pb Ma) & Gold-beige, mosaic gold & Clothing, bird \\
& & Red, vermilion & Writing ink \\
& & Green, orpiment and indigo & Landscape \\
$\mathrm{K}$ & $\mathrm{Ka}(3.169-3.406)$ & Gold-beige, together with mosaic gold Sn, S & Clothing, bird \\
& & Red, brazilwood lake & Clothing \\
$\mathrm{Ca}$ & $\mathrm{Ka}(3.554-3.821)$ & Red, brazilwood lake & Clothing \\
& & Pink, gypsum & ground layer for leaf gilding \\
$\mathrm{Fe}$ & $\mathrm{Ka}(6.249-6.485)$ & Flesh tone, not identified & Falconers incarnate \\
& & Brown, iron gall ink & Writing ink \\
& $\mathrm{Ka}(7.877-8.144)$ & Color unclear, together with azurite Cu & Component in blue color \\
$\mathrm{Cu}$ & $\mathrm{La}(3.411-3.484)$ & Blue, azurite & Background color, decoration \\
$\mathrm{Sn}$ & $\mathrm{La}(9.572-9.836)$ & Gold-beige, mosaic gold & Clothing, bird \\
$\mathrm{Au}$ & $\mathrm{La}(10.216-10.720)$ & Gold & Gold leaf \\
$\mathrm{Hg}$ & & Pink, vermilion & Component of ground layer \\
& & Red, vermilion & Writing ink \\
$\mathrm{Pb}$ & $\mathrm{La}(10.216-10.720)$ & Red, minium & Clothing, decoration \\
& & Pink, lead white mixed with brazilwood & Initial letter
\end{tabular}

indication for a different authorship, so far only assumed by means of stylistic differences.

However, some interesting questions remain unclear, in particular the precise characterization of the gold beige pigment containing $\mathrm{Sn}, \mathrm{S}$ and $\mathrm{K}$, and also the red Brazilwood lake. Both have not received our increased interest until the evaluation of the data from the second campaign. Unfortunately, no Raman analyses could be performed, which possibly would have enabled a clear identification. This demonstrates the need for improved evaluation tools for the complex spectra obtained from multiple non-invasive analyses, particularly if the access to the studied objects is limited. With respect to the available time for analysis, a microscopic investigation prior to the instrumental analyses probably would have allowed to utilize the available methods more efficient. Moreover, the rFTIR single point measurement detection of a proteinaceous material over the gold leaf, as well as calcium soaps in certain colors raised the question about the distribution of these materials in order to be able to explain the application in the miniature painting technique of the thirteenth century. This strongly indicates an urgent demand for mobile rFTIR scanning equipment, which can be used in libraries or museums.

XRF scanning provided valuable information about the painting technique, although the scans are quite time consuming. In contrast to single point measurements, the risk of misinterpretation of XRF results due to material inhomogeneities is much lower for scans, where the information of multiple measurements is gathered and hence a much clearer picture of the materials can be obtained. This particularly accounted for the gold-beige color, where the detection of $\mathrm{Sn}$ and $\mathrm{Pb}$ in the single point measurements would rather have argued for the use of lead tin yellow than of mosaic gold. On the other hand, the scanning results showed that the short measurement times for each point may limit the detection of elements present in small quantities, as it was the case for As in the presented study. This clearly shows the importance of single point measurements with longer acquisition times in the areas intended to scan in order to prevent misinterpretation of scan data.

\footnotetext{
Abbreviations

CIMA: Centre of Image and Material Analysis in Cultural Heritage; FORS: Fiber optic reflectance spectroscopy; HRSM: Hochschul-Raum Struktur-Mittel; IRUG : Infrared and Raman Users Group; mp: Measurement point; r: Recto; rFTIR: Fourier transform infrared spectroscopy in the reflection mode; v: Verso; XRF: X-ray fluorescence.
} 


\section{Supplementary Information}

The online version contains supplementary material available at https://doi. org/10.1186/s40494-021-00553-w.

Additional file 1 Vienna Moamin, folio 1r. The analyzed points are indicated with white arrowheads.

Additional file 2: Vienna Moamin, folio 10v. The analyzed points are indicated with white arrowheads.

Additional file 3: Vienna Moamin, folio 19r. The analyzed points are indicated with white arrowheads.

Additional file 4: Vienna Moamin, folio 37r. The analyzed points are indicated with white arrowheads.

Additional file $\mathbf{5}$ Vienna Moamin, folio 47v. The analyzed points are indicated with white arrowheads.

Additional file 6 Self-built fiber optic probe: a) collimator $0.3 \mathrm{~mm}$ (latex), b) $0^{\circ} / 45^{\circ}$-component, c) xz-axial positioning mechanism, d) laser pointer for precise positioning, e) battery compartment, and f) switch for lasers on/off.

Additional file $\mathbf{7}$ Comparison of the absorption index spectrum of a light blue colorant on folio $37 \mathrm{r}$ (red) with the IRUG reference spectra (obtained in transmission mode) azurite MP00001 (blue) and lead white MP00107 (yellow).

Additional file $\mathbf{8}$ Left: Comparison of the Raman spectrum of a green colorant on folio $47 \mathrm{v}$ (red) with reference spectra of orpiment (yellow) and indigo (blue). Right: FORS spectrum from the same measurement point in comparison with an indigo reference.

Additional file $\mathbf{9}$ Comparison of the Raman spectrum of a brown colorant on folio $1 r$ (red) with an iron oxide brown reference (blue)

Additional file 10 Comparison of the Raman spectrum of a black colorant on folio 10v (red) with a carbon black reference (blue).

Additional file 11 Comparison of the absorption index spectrum of a blue colorant on folio $1 \mathrm{r}$ (red) with the IRUG reference spectrum (obtained in transmission mode) calcium stearate OF00108 (blue).

Additional file 12: The original size of the image stitched in Photoshop in Fig. 11 is $3355 \times 3355$ px.

\section{Acknowledgements}

The authors wish to thank Dr. Matthias Pfaffenbichler and Dr. Stefan Krause (Collection of Arms and Armour, Kunsthistorisches Museum Wien) who provided access to the Manuscript, and furthermore, Mag. Petra Fuchs (Conservator) for her support. A special thanks to Prof. Baudouin Van den Abeele (Université Catholique de Louvain-UCLouvain) for the valuable information about the provenance and history of the Vienna Moamin.

\section{Authors' contributions}

WV performed the rFTIR and FORS analyses, carried out the interpretation of the results, and drafted the manuscript. XRF and Raman analyses, as well as the interpretation of the results were carried out by BF and FC. MS participated in the coordination of the study, and helped to draft the manuscript. All authors read and approved the final manuscript.

\section{Funding}

The Austrian Science Fund (FWF) and the Austrian Ministry for Science, Research and Economy (BMWFW) are gratefully acknowledged for the financial supports: P29892 as well as Hochschulraum-Strukturmittelfonds (HRSM)-project "Analysis and Conservation of Cultural Heritage-Modern Imaging and Material Analysis Methods for the Visualization, Documentation and Classification of Historical Written Material (Manuscripts)".

\section{Availability of data and materials}

\section{Declarations}

Competing interests

The authors declare that they have no competing interest.

Received: 28 December 2020 Accepted: 15 June 2021

Published online: 23 July 2021

\section{References}

1. Georges S. Das zweite Falkenbuch Kaiser Friedrichs II. Quellen, Entstehung, Überlieferung und Rezeption des Moamin, mit einer Edition der lateinischen Überlieferung, De Gruyter, Berlin. 2008. https://doi.org/10. $1524 / 9783050049816$.

2. Van den Abeele B. The Moamin, an Arabic treatise on falconry translated for the Emperor Frederick II of Hohenstaufen in 1240. Int J Falconry. 2018;10:64-9.

3. Van den Abeele B. Le Moamin illustré de Vienne (circa 1300): les soins des oiseaux maladies. Commentaria Classica. 2018. 5:427-445 (online). http:// www.commentariaclassica.altervista.org/vandenabeele.pdf. Accessed 7 Dec 2020.

4. https://www.adeva.com/f/805/The-Vienna-Moamin---Real-Gold-Edition. Accessed 7 Dec 2020

5. Van den Abeele B. Der Wiener Moamin. Kommentar zur FaksimileAusgabe. Akademische Druck- u. Verlagsanstalt, Graz/Austria. 2021. ISBN: 978-3-201-02049-7. In press (20.4.2021).

6. CIMA is an interuniversity research institution with an interdisciplinary approach aiming at the investigation of cultural heritage and was founded in 2014, please see https://cima.or.at/. Accessed 7 Dec 2020.

7. Chaplin TD, Clark RJH, Jones R, Gibbs R. Pigment analysis by Raman microscopy and portable X-ray fluorescence (pXRF) of thirteenth to fourteenth century illuminations and cuttings from Bologna. Phil Trans R Soc A. 2016:374:20160043. https://doi.org/10.1098/rsta.2016.0043.

8. Trentelman K, Turner N. Investigation of the painting materials and techniques of the late-15th century manuscript illuminator Jean Bourdichon. J Raman Spectrosc. 2009;40:577-84. https://doi.org/10.1002/jrs.2186.

9. Ricciardi P, Mazzinghi A, Legnaioli S, Ruberto C, Castelli L. The Choir Books of San Giorgio Maggiore in Venice: results of in depth non-invasive analyses. Heritage. 2019;2:1684-701. https://doi.org/10.3390/heritage2020103.

10. Legrand S, Ricciardi P, Nodari L, Janssens K. Non-invasive analysis of a 15th century illuminated manuscript fragment: point-based vs imaging spectroscopy. Microchem J. 2018;138:162-72.

11. de Viguerie L, Rochut S, Alfeld M, Walter P, Astier S, Gontero V, Boulc'h F. $\mathrm{XRF}$ and reflectance hyperspectral imaging on a 15th century illuminated manuscript: combining imaging and quantitative analysis to understand the artist's technique. Herit Sci. 2018;6:11. https://doi.org/10.1186/ s40494-018-0177-2.

12. Manukyan KV, Raddell M, Sestak E, Gura DT, Zachary D Schultz ZD, Wiescher M. Pigment and ink analysis of medieval books through complementary spectroscopy techniques. Glob J Arch Anthropol. 2018;3(4): GJAA.MS.ID.555619. https://doi.org/10.19080/GJAA.2018.03.555619.

13. Legrand S, Alfeld M, Vanmeert F, De NolfW, Janssens K. Macroscopic Fourier transform infrared scanning in reflection mode (MA-rFTIR), a new too for chemical imaging of cultural heritage artefacts in the mid-infrared range. Analyst. 2014;139:2489-98. https://doi.org/10.1039/c3an02094k.

14. Price BA, Pretzel B, Lomax SQ, editors. Infrared and Raman users group spectral database. 2007 ed. vol. 1, 2.

15. Vetter W. Charakterisierung von Pigment/Bindemittel-Systemen im Bereich der Kunst mittels FTIR- und UVNis/NIR-Spektroskopie unter besonderer Berücksichtigung zerstörungsfreier Methoden. Doctoral thesis. TU Wien. 2014. https://www.ub.tuwien.ac.at/diss/AC11719923.pdf. Accessed 10 Apr 2021.

16. Nabais P, Castro R, Videira Lopes G, Correia de Sousa L, Melo MJ. Singing with light: an interdisciplinary study on the medieval Ajuda Songbook. J Medieval Iberian Stud. 2016;8(2):283-312. https://doi.org/10.1080/17546 559.2016.1234061. 
17. Samari M, Ridha F, Manovic V, Macchi A, Anthony EJ. Direct capture of carbon dioxide from air via lime-based sorbents. Mitig Adapt Strateg Global Change. 2020;25:25-41. https://doi.org/10.1007/s11027-019-9845-0.

18. Gunasekaran S, Anbalagan G, Pandi S. Raman and infrared spectra of carbonates of calcite structure. J Raman Spectrosc. 2006;37:892-9. https:// doi.org/10.1002/jrs.1518.

19. Smith SB, Gill CA, Lunt DK, Brooks MA. Regulation of fat and fatty acid composition in beef cattle. Asian-Aust J Anim Sci. 2009;22(9):1225-33. https://doi.org/10.5713/ajas.2009.r.10.

20. Otero V, Sanches D, Montagner C, Vilarigues M, Carlyle L, Lopes JA, Melo MJ. Characterisation of metal carboxylates by Raman and infrared spectroscopy in works of art. J Raman Spectrosc. 2014;45:1013-8. https://doi. org/10.1002/jrs.4520.

21. Gönen M, Öztürk S, Balköse D, Okur S, Ülkü S. Preparation and characterization of calcium stearate powders and films prepared by precipitation and Langmuir-Blodgett techniques. Ind Eng Chem Res. 2010;49:1732-6. https://doi.org/10.1021/ie901437d.

22. Lee AS, Mahon PJ, Creagh DC. Raman analysis of iron gall inks on parchment. Vib Spectrosc. 2006;41:170-5. https://doi.org/10.1016/j.vibspec. 2005.11.006.

23. Lee AS, Otieno-Alego V, Creagh DC. Identification of iron-gall inks with near-infrared Raman microspectroscopy. J Raman Spectrosc 2008;39:1079-84. https://doi.org/10.1002/jrs.1989.

24. Díaz Hidalgo RJ, Córdoba R, Nabais P, Silva V, Melo MJ, Pina F, Teixeira N, Freitas $V$. New insights into iron-gall inks through the use of historically accurate reconstructions. Herit Sci. 2018;6:63. https://doi.org/10.1186/ s40494-018-0228-8.

25. Aceto M, Calà E. Analytical evidences of the use of iron-gall ink as a pigment on miniature paintings. Spectrochim Acta A Mol Biomol Spectrosc. 2017;187:1-8. https://doi.org/10.1016/j.saa.2017.06.017.

26. Boyatzis SC, Velivasaki G, Malea E. A study of the deterioration of aged parchment marked with laboratory iron gall inks using FTIR-ATR spectroscopy and micro hot table. Herit Sci. 2016;4:13. https://doi.org/10.1186/ s40494-016-0083-4.

27. Monico L, Rosi F, Miliani C, Daveri A, Brunetti BG. Non-invasive identification of metal-oxalate complexes on polychrome artwork surfaces by reflection mid-infrared spectroscopy. Spectrochim Acta A Mol Biomol Spectrosc. 2013;166:270-80. https://doi.org/10.1016/j.saa.2013.06.084.

28. Ferrer N, Sistach MC. Analysis of sediments on iron gall inks in manuscripts. Restaurator. 2013;34(3):175-93. https://doi.org/10.1515/ res-2013-0010.

29. Vahur S, Knuutinen U, Leito I. ATR-FT-IR spectroscopy in the region of $500-230 \mathrm{~cm}^{-1}$ for identification of inorganic red pigments. Spectrochim Acta A Mol Biomol Spectrosc. 2009;73:764-71. https://doi.org/10.1016/j. saa.2009.03.027

30. Vetter W, Latini I, Schreiner M. Azurite in medieval illuminated manuscripts: a reflection-FTIR study concerning the characterization of binding media. Herit Sci. 2019;7:21. https://doi.org/10.1186/s40494-019-0262-1.

31. Aru M, Burgio L, Rumsey MS. Mineral impurities in azurite pigments: artistic or natural selection? J Raman Spectrosc. 2014;45:1013-8. https:// doi.org/10.1002/jrs.4469.

32. Siidra O, Nekrasova D, Depmeier W, Chukanov N, Zaitsev A, Turner N. Hydrocerussite-related minerals and materials: structural principles, chemical variations and infrared spectroscopy. Acta Cryst. 2018;B74:18295. https://doi.org/10.1107/S2052520618000768.

33. Eastaugh N, Walsh V, Chaplin T, Siddall R. Pigment compendium, a dictionary and optical microscopy of historical pigments. Oxford: Butterworth-Heinemann; 2008. p. 667. ISBN: 978-0-7506-8980-9.

34. West Fitzhugh E. Orpiment and Realgar. In: West Fitzhugh E, editor. Artists' pigments, a handbook of their history and characteristics. Vol. 3. London Archetype Publications; 1997. p. 54. ISBN: 978-1-904982-76-0.

35. Prasad PSR, Chaitanya VK, Prasad KS, Rao DN. Direct formation of the $\gamma-\mathrm{CaSO}_{4}$ phase in dehydration process of gypsum: in situ FTIR study. Am Miner. 2005:90:672-8. https://doi.org/10.2138/am.2005.1742.

36. Griffiths PR, de Haseth JA. Specular reflection. In: Fourier transform infrared spectroscopy. Second edn. Hoboken, New Jersey: Wiley. 2007; p. 277 ff. ISBN: 978-0-471-19404-0.

37. Grygar T, Hradilová J, Hradil D, Bezdička P, Bakardjieva S. Analysis of earthy pigments in grounds of Baroque paintings. Anal Bioanal Chem. 2003:375:1154-60. https://doi.org/10.1007/s00216-002-1708-x.
38. Gil M, Carvalho ML, Seruya A Candeias AE, Mirao J, Queralt I Yellow and red ochre pigments from southern Portugal: elemental composition and characterization by WDXRF and XRD. Nucl Instrum Meth A. 2007;580:72831. https://doi.org/10.1016/j.nima.2007.05.131.

39. Vaculikova L, Plevova E, Vallova S, Koutnik I. Characterization and differentiation of kaolinites from selected Czech deposits using infrared spectroscopy and differential thermal analysis. Acta Geodyn Geomater. 2011;8(1):59-67.

40. Nodari L, Ricciardi P. Non-invasive identification of paint binders in illuminated manuscripts by ER-FTIR spectroscopy: a systematic study of the influence of different pigments on the binders' characteristic spectral features. Herit Sci. 2019;7:7. https://doi.org/10.1186/s40494-019-0249-y.

41. Ross JL. A note on the use of mosaic gold. Stud Conserv. 1973;18:174-6.

42. Thompson DV. The materials and techniques of medieval paintings. New York: Dover Publications; 1956. p. 181-184. ISBN: 0-486-20327-1.

43. Melo MJ, Otero V, Vitorino T, Araujo R, Muralha VSF, Lemos A, Picollo M. A spectroscopic study of Brazilwood paints in medieval books of hours. Appl Spectrosc. 2014;68(4):434-44. https://doi.org/10.1366/13-07253.

44. Burton LA, Whittles TJ, Hesp D, Linhart WM, Skelton JM, Hou B, Webster RF, O'Dowd G, Reece C, Cherns D, Fermin DJ, Veal TD, Dhanak VR, Walsh A. Electronic and optical properties of single crystal $\mathrm{SnS}_{2}$ : an earth-abundant disulfide photocatalyst. J Mater Chem A. 2016;4:1312-8. https://doi. org/10.1039/c5ta08214e.

45. Voznyi A, Kosyak V, Onufrijevs P, Grase L, Vecstaudža J, Opanasyuk A, Medvid $A$. Laser-induced $\mathrm{SnS}_{2}$-SnS phase transition and surface modification in $\mathrm{SnS}_{2}$ thin films. J Alloys Compd. 2016;688:130-9. https://doi.org/10. 1016/j.jallcom.2016.07.103.

46. Aceto M, Agostino A, Fenoglio G, Idone A, Gulmini M, Picollo M, Ricciardi $P$, Delaney JK. Characterisation of colourants on illuminated manuscripts by portable fibre optic UV-visible-NIR reflectance spectrophotometry. Anal Methods. 2014:6:1488-500. https://doi.org/10.1039/c3ay41904e.

47. Schmidt CM, Trentelman KA. 1064 nm dispersive Raman micro-spectroscopy for the in-situ identification of organic red colorants. e-PS. 2009;6:10-21.

48. Mulholland R, Howell D, Beeby A, Nicholson CE, Domoney K. Identifying eighteenth century pigments at the Bodleian library using in situ Raman spectroscopy, XRF and hyperspectral imaging. Herit Sci. 2017;5:43. https://doi.org/10.1186/s40494-017-0157-y.

49. Coccato A, Jehlicka J, Moens L, Vandenabeele P. Raman spectroscopy for the investigation of carbon-based black pigments. J Raman Spectrosc. 2015;46:1003-15. https://doi.org/10.1002/jrs.4715.

50. Thompson DV. The materials and techniques of medieval paintings. New York: Dover Publications; 1956. p. 50-58. ISBN: 0-486-20327-1.

51. Kroustallis S. Binding media in medieval manuscript illumination: a source research. In: Medieval Colours: between beauty and meaning. Rev Hist Arte Sér W. 201;1:112-125. https://revistadehistoriadaarte.files.wordpress. com/2011/09/art08.pdf. Accessed 10 Apr 2021.

52. Griffiths PR, de Haseth JA. Specular reflection. In: Fourier transform infrared spectrometry. 2nd edn. Hoboken, New Jersey: Wiley. 2007; p. 297. ISBN: 978-0-471-19404-0.

53. Vieira M, Nabais $P$, Angelin EM, Araújo R, Lopes JA, Martín L, Sameño M, Melo MJ. Organic red colorants in Islamic manuscripts (12th-15th c.) produced in al-Andalus, part 1. Dyes Pigm. 2019;166:451-9. https://doi. org/10.1016/j.dyepig.2019.03.061.

54. Thompson DV. The materials and techniques of medieval paintings. New York: Dover Publications; 1956. p. 100 and 174. ISBN: 0-486-20327-1.

55. Eastaugh N, Walsh V, Chaplin T, Siddall R. Pigment compendium, a dictionary and optical microscopy of historical pigments. Oxford: Butterworth-Heinemann; 2008. p. 274. ISBN: 978-0-7506-8980-9.

\section{Publisher's Note}

Springer Nature remains neutral with regard to jurisdictional claims in published maps and institutional affiliations. 